\title{
The submillimeter spectrum of deuterated glycolaldehydes ${ }^{\star}$
}

\author{
A. Bouchez ${ }^{1,2,3}$, L. Margulès ${ }^{3}$, R. A. Motiyenko ${ }^{3}$, J.-C. Guillemin ${ }^{4}$, A. Walters ${ }^{1,2}$, S. Bottinellii ${ }^{1,2}$, \\ C. Ceccarelli ${ }^{5}$, and C. Kahane ${ }^{5}$ \\ 1 Université de Toulouse, UPS-OMP, IRAP, Toulouse, France \\ e-mail: abouchez@irap.omp.eu \\ 2 CNRS, IRAP, 9 Av. colonel Roche, BP 44346, 31028 Toulouse Cedex 4, France \\ ${ }^{3}$ Laboratoire de Physique des Lasers, Atomes, et Molécules, UMR CNRS 8523, Université de Lille I, 59655 Villeneuve d'Ascq \\ Cedex, France \\ ${ }^{4}$ Sciences Chimiques de Rennes, École Nationale Supérieure de Chimie de Rennes, CNRS, UMR 6226, Avenue du Général Leclerc, \\ CS 50837, 35708 Rennes Cedex 7, France \\ 5 IPAG, Université Joseph Fourier, CNRS, BP 53, 38041 Grenoble Cedex 9, France
}

Received 21 December 2011 / Accepted 16 February 2012

\begin{abstract}
Context. Glycolaldehyde, a sugar-related interstellar prebiotic molecule, has recently been detected in two star-forming regions, Sgr $\mathrm{B} 2(\mathrm{~N})$ and $\mathrm{G} 31.41+0.31$. The detection of this new species increased the list of complex organic molecules detected in the interstellar medium (ISM) and adds another level to the chemical complexity present in space. Besides, this kind of organic molecule is important because it is directly linked to the origin of life. For many years, astronomers have been struggling to understand the origin of this high chemical complexity in the ISM. The study of deuteration may provide crucial hints.

Aims. In this context, we have measured the spectra of deuterated isotopologues of glycolaldehyde in the laboratory: the three monodeuterated ones $\left(\mathrm{CH}_{2} \mathrm{OD}-\mathrm{CHO}, \mathrm{CHDOH}-\mathrm{CHO}\right.$ and $\left.\mathrm{CH}_{2} \mathrm{OH}-\mathrm{CDO}\right)$ and one dideuterated derivative (CHDOH-CDO) in the ground vibrational state.

Methods. Previous laboratory work on the D-isotopologues of glycolaldehyde was restricted to less than $26 \mathrm{GHz}$. We used a solidstate submillimeter-wave spectrometer in Lille with an accuracy for isolated lines better than $30 \mathrm{kHz}$ to acquire new spectroscopic data between 150 and $630 \mathrm{GHz}$ and employed the ASFIT and SPCAT programs for analysis.

Results. We measured around 900 new lines for each isotopologue and determined spectroscopic parameters. This allows an accurate prediction in the ALMA range up to $850 \mathrm{GHz}$.

Conclusions. This treatment meets the needs for a first astrophysical research, for which we provide an appropriate set of predictions.
\end{abstract}

Key words. ISM: molecules - methods: laboratory - submillimeter: ISM - molecular data - line: identification

\section{Introduction}

A search for various major isotopologues of key species in the interstellar medium (ISM) is still hampered by a scarcity of reliable spectroscopic data from laboratory measurements, which has also been the case for deuterated glycolaldehyde. However, studying the spectra and abundances of isotopologues in the ISM can be a valuable tool in chemical and physical modelling. In particular, a study of the isotopologues of known organic molecules can provide information for understanding molecular complexity in star-forming regions (SFRs). The detection of deuterated molecules has a strong impact for understanding and modelling the formation and deuteration process of molecules. This is especially interesting for complex molecules for which it may allow differentiating the gas-phase and solid-state formation pathways.

Glycolaldehyde is the smallest possible molecule that contains both an aldehyde group and a hydroxyl group and this dimer of formaldehyde can be considered as the simplest sugar.

* Full Table 2 and Tables 3-5 are only available in electronic form at the CDS via anonymous ftp to

cdsarc.u-strasbg.fr $(130.79 .128 .5)$ or via

http://cdsarc.u-strasbg.fr/viz-bin/qcat?J/A+A/540/A51
It is an isomer of methyl formate $\left(\mathrm{HCOOCH}_{3}\right)$ and acetic acid $\left(\mathrm{CH}_{3} \mathrm{COOH}\right)$, and the relative abundance of these isomers are two of the three exceptions of the minimum energy principle (Lattelais et al. 2009); the most stable isomer, acetic acid, should be the most abundant, but in all astronomical sources where it was searched for, methyl formate is much more abundant, and acetic acid and glycolaldehyde have about the same abundance. These exceptions could be due to the kinetic or abundance effect, or to the existence of different routes of formation with no intermediate in common or to specific depletion on the grains of one isomer with respect to the others.

Glycolaldehyde is a probable prebiotic molecule and a key intermediate in the formose reaction, i.e. the formation from formaldehyde of sugars containing three, four, or five C-atoms. It has been detected in the SFR Sgr B2(N) by Hollis et al. (2000) from 2 and $3 \mathrm{~mm}$ single-dish surveys, with an estimated rotational temperature $\left(T_{\text {rot }}\right)$ of $35 \mathrm{~K}$ from 34 lines detected. Beltrán et al. (2009) reported identification of three lines towards the hot core G31.41 +0.31 using the Plateau de Bure Interferometer. Their analysis indicated that emission comes from the hottest $(\geq 300 \mathrm{~K})$ and the densest $\left(\geq 2 \times 10^{8} \mathrm{~cm}^{-3}\right)$ region closest to the protostar. Although some transitions of the parent molecule have been observed, no investigation of 
the isotopologues has yet been reported because of (i) the lack of spectroscopic data and (ii) the expected weakness of their emission. However, observations like this would help us to understand the formation pathway of the parent molecules, which is currently unclear. Indeed, several gas-phase and solidphase routes of formation of glycolaldehyde have been proposed. The possible gas-phase pathway is the formose reaction (Jalbout et al. 2007): protonated formaldehyde $\mathrm{CH}_{2} \mathrm{OH}^{+}$can react with formaldehyde to yield glycoladehyde. However, a recent study by Simakov et al. (2011) compromises this formation route: the authors reported that protonated formaldehyde does not lead to protonated glycolaldehyde. Other recent studies (e.g. Hamberg et al. 2010) have shown that dissociative recombination reactions of a protonated molecule $\mathrm{HX}^{+}$do not lead to the stable molecule $\mathrm{X}$ for the most part. Hence a more probable route of formation is via the reaction of methanol and carbon monoxide on interstellar ices (Bennett \& Kaiser 2007):

$$
\mathrm{CH}_{3} \mathrm{OH}+\mathrm{CO} \rightarrow \mathrm{CH}_{2} \mathrm{OH}+\mathrm{HCO} \rightarrow \mathrm{OHCH}_{2}-\mathrm{CHO} \text {. }
$$

To date, no identification of the deutero-isotopologues has been reported, but a comparison of $\mathrm{D} / \mathrm{H}$ ratios may be valuable for understanding the formation of glycolaldehyde. Indeed, in hot cores and hot corinos the deuteration fraction of complex organic molecules is much greater than the cosmic $\mathrm{D} / \mathrm{H}$ ratio $\left(10^{-5}\right.$; Oliveira \& Hébrard 2006) because it is around $10^{-3}$. This ratio is more studied in hot corinos where the process of deuteration is considered to be overabundant (detected molecules can be doubly or triply deuterated, as described for example by Ceccarelli et al. 1998). This is used to determine the formation pathway as shown by Cazaux et al. (2011) and Coutens et al. (2012), with the formation of water and formaldehyde. However, the process could be similar for hot cores, as shown by Turner (1990) or recently by Ratajczak et al. (2011). Therefore we recently made extensive laboratory measurements to be able to search for the millimeter-wave spectrum of D-isotopologues of glycolaldehyde in the ISM. These measurements are particularly relevant to ALMA because of its high sensitivity and high spatial resolution (that could, for example, reduce the spectral line confusion). However, it might also be possible to identify these isotopologues using sensitive single-dish instruments such as the IRAM-30 m.

The first microwave spectrum measurements of the main isotopologue of glycolaldehyde were reported by Marstokk \& Møllendal (1970), who obtained the pure rotational and centrifugal distortion constants of the ground state and three vibrational excited states, as well. In addition, in 1973 they were able to determine the electric dipole moment as $\mu_{b}=2.33 \mathrm{D}$ and $\mu_{a}=0.26 \mathrm{D}$. Then Butler et al. (2001) extended millimeter and submillimeter assignments for the ground vibrational state from 128 to $354 \mathrm{GHz}$. A subsequent study by Widicus Weaver et al. (2005) included assignments for the ground state and three excited vibrational states up to $354 \mathrm{GHz}$ as well as a partition function analysis. Finally, Carroll et al. (2010) reported the spectrum of the ground vibrational state in selected regions of the submillimeter region up to $1.2 \mathrm{THz}$. Less information is available on the other isotopologues of glycolaldehyde. The only published measurements for the deuterium isotopologues are from Marstokk \& Møllendal (1971), whose measurements were restricted to between 12 and $26 \mathrm{GHz}$. Considering the importance of understanding the formation of this molecule and the development of (sub)millimeter observatories, new data are needed, which is why we measured the spectra of all mono-deuterated isotopologues $\left(\mathrm{CH}_{2} \mathrm{OD}-\mathrm{CHO}, \mathrm{CHDOH}-\mathrm{CHO}, \mathrm{CH}_{2} \mathrm{OH}-\mathrm{CDO}\right.$,

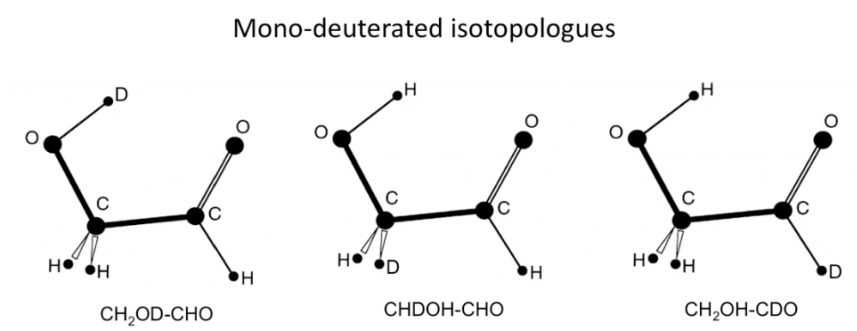

Doubly deuterated isotopologues

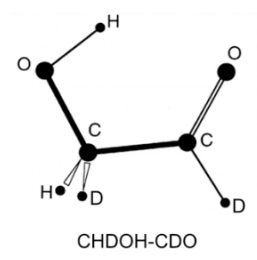

Fig. 1. Representation of all mono-deuterated isotopologues of glycolaldehyde and the doubly deuterated one.

cf. Fig. 1) of glycolaldehyde and one doubly deuterated one (CHDOH-CDO, cf. Fig. 1) between 150 and $630 \mathrm{GHz}$ in the laboratory.

\section{Experiments}

The submillimeter-wave measurements (150-630 GHz) were performed using the Lille spectrometer (Motiyenko et al. 2010) based only on solid-state sources. The frequency of an Agilent synthesizer E8257D (12.5-17.5 GHz) was first multiplied by six and amplified by a Spacek active sextupler, providing an output power of $+15 \mathrm{dBm}$ in the $W$-band range $(75-110 \mathrm{GHz})$. This power is high enough to use passive Schottky multipliers $(\times 2$, $\times 3, \times 5, \times 3 \times 2$ ) from Virginia Diodes Inc in the next stage of the frequency multiplication chain. As a detector we used an InSb liquid He-cooled bolometer from QMC Instruments Ltd. To improve the sensitivity of the spectrometer, the sources were frequency modulated at $10 \mathrm{kHz}$ and phase-sensitive detection at $2 \mathrm{f}$ was employed. The absorption cell is a stainless-steel tube $(6 \mathrm{~cm}$ diameter, $220 \mathrm{~cm}$ long). The sample pressure during measurements was about 1.5 Pa (15 $\mu$ bar) and the linewidth was limited by Doppler broadening. Measurements were performed at room temperature. The accuracy of the measurements for isolated lines is estimated to be better than $30 \mathrm{kHz}$. However, if the lines were blended or had a poor signal-to-noise ratio, they were given a weight of 100 or even $200 \mathrm{kHz}$.

The synthesis procedure was as follows:

Synthesis of deuterated glycolaldehydes: 1,4-Dioxane-2,5-diol and dihydroxyfumaric acid hydrate were purchased from Aldrich and used without additional purification.

Synthesis of $\mathrm{DOCH}_{2} \mathrm{CHO}$ : 1,4-Dioxane-2,5-diol (5 g) was dissolved in deuterated water $(10 \mathrm{~mL})$ and heated to $70{ }^{\circ} \mathrm{C}$ for $1 \mathrm{~h}$. The mixture was lyophylisated to obtain a solid sample of $\mathrm{DOCH}_{2} \mathrm{CHO}$ with about $25 \%$ isotopic purity.

Synthesis of HOCHD-CHO, $\mathrm{HOCH}_{2}-\mathrm{CDO}$ and HOCHDCDO: The synthesis reported by Marstokk \& Møllendal (1973) was used starting from dihydroxyfumaric acid hydrate dissolved in about $80 \% \mathrm{D}_{2} \mathrm{O}$. Decarboxylation in dry pyridine as described by Powers et al. gave an about 2:1:1 


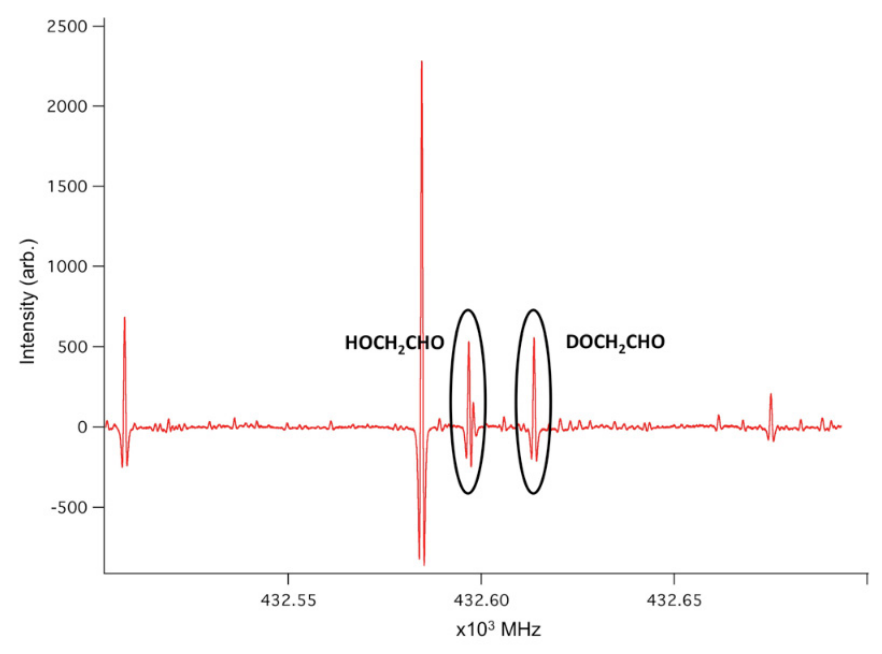

Fig. 2. Part of the spectrum of the first sample containing $\mathrm{HOCH}_{2} \mathrm{CHO}$ (transition $39_{7,32}-38_{8,31}$ and intensity $\alpha=1.37 \times 10^{-4} \mathrm{~cm}^{-1}$ ) in the first excited vibrational state and $\mathrm{DOCH}_{2} \mathrm{CHO}$ (transition $38_{7,32}-37_{6,31}$ and intensity $\alpha=5.83 \times 10^{-4} \mathrm{~cm}^{-1}$ ).

mixture of $\mathrm{HOCHD}-\mathrm{CHO}, \mathrm{HOCH}_{2}-\mathrm{CDO}$ and $\mathrm{HOCHD}-$ CDO. Small amounts of residual pyridine, a compound more volatile than glycolaldehyde, led to relatively intense signals in the microwave spectrum.

\section{Analysis}

Glycolaldehyde is a prolate asymmetric top molecule $(\kappa=$ -0.7). Therefore we used the Watson A-reduction Hamiltonian in the $I^{r}$ representation in the analysis of its spectra. Spectral data were fitted using the ASFIT programme ${ }^{1}$. Predictions were made using the SPCAT programme (Pickett et al. 1998) and the parameter set resulting from ASFIT and the dipole moments determined by Marstokk \& Møllendal (1973).

The first species measured and analysed was $\mathrm{DOCH}_{2} \mathrm{CHO}$ with the enriched sample containing only this isotopologue and the parent species with an estimated proportion of $25 \%$ of OD as shown in Fig. 2.

Initial predictions were made using lines reported in Marstokk \& Møllendal (1971) between 12 and 26 GHz. First, we identified the strong R-branch transitions with $K_{a}=0$ and 1 between 150 and $310 \mathrm{GHz}$ up to $J=31$. The low $\mathrm{J}$ lines were easily recognised doublets ( $K_{a}$ selection rules: $\left.1-0 \& 0-1\right)$ with systematic decrease in the split towards higher $\mathrm{J}$ and visible addition of the intensities as the components merge. All transitions were shifted systematically from the predictions up to a maximum of around $10 \mathrm{MHz}$ at $J=20$. The newly identified lines were then included in the fit. Next we identified lines by systematically increasing $K_{a}$ up to $K_{a}^{\prime}=11$ and finally measured all other identifiable R-branch lines (up to $J=59$ and $K_{a}^{\prime}=18$ ). We then identified three $\mathrm{Q}$ branches and assigned all lines possible. The same procedure was then repeated for a second frequency band between 400 and $630 \mathrm{GHz}$. Finally, we assigned some P-branch transitions. Since these lines were much weaker, assignment was only possible in the region around $300 \mathrm{GHz}$ where the spectrometer is most sensitive. Because the strong $b$-component of the dipole moment $(2.33 \mathrm{D})$ is much stronger

\footnotetext{
${ }_{1}$ Kisiel, PROSPE, http://www.ifpan.edu.pl/ kisiel/ prospe.htm
}

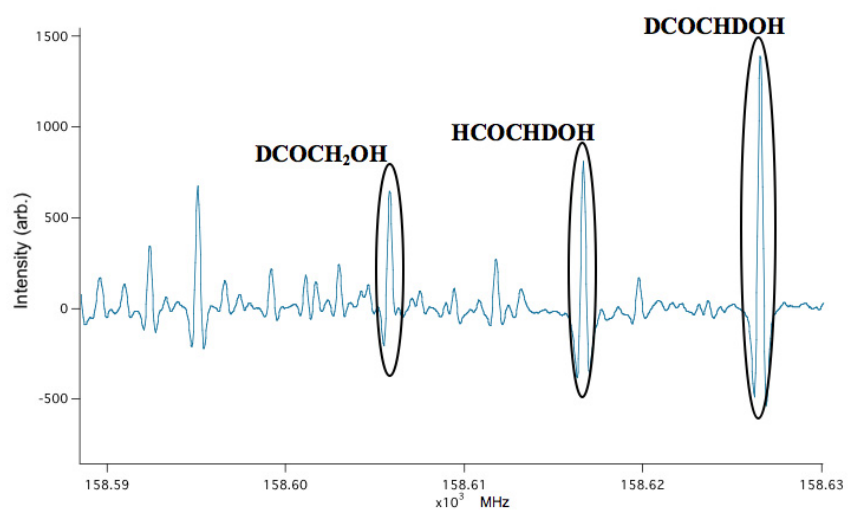

Fig. 3. Part of the spectrum of the second sample containing $\mathrm{CHDOH}-$ CHO (transition $23_{3,20}-23_{2,21}$ and intensity $\alpha=1.62 \times 10^{-4} \mathrm{~cm}^{-1}$ ), $\mathrm{CH}_{2} \mathrm{OH}-\mathrm{CDO}$ (transition $74,3-6_{3,4}$ and intensity $\alpha=1.90 \times 10^{-4} \mathrm{~cm}^{-1}$ ) and $\mathrm{CHDOH}-\mathrm{CDO}$ (transition $33_{10,23}-33_{9,22}$ and intensity $\alpha=2.01 \times$ $\left.10^{-4} \mathrm{~cm}^{-1}\right)$.

than the $a$-component $(0.26 \mathrm{D})$, all newly assigned lines are $b$-type transitions.

To check the relative weight of the Lille and centimeterwave data sets, the method of iteratively reweighted leastsquares (IRLSQ) of Margules et al. (2010) was used. As a result, 63 of the 65 lines from Marstokk \& Møllendal (1971) were rejected from the fit. We found a systematic deviation of $150 \mathrm{kHz}$ for these measurements, and consequently they were excluded from the final fit. The same kind of deviation was also found for the data on other deuterated species available from Marstokk \& Møllendal (1971). The final fit is given in the supplementary Table 2 . It contains 889 new identified lines (1433 transitions, because there are 355 unresolved doublets and 63 quadruplets) up to $J=64$ and $K_{a}^{\prime}=37$.

We were able to determine all sextic centrifugal distortion parameters. Including some octic parameters made no significant improvement and consequently they were excluded from the final fit. The rms deviation of the fit is $27 \mathrm{kHz}$ with a weighted rms of 0.90 , which indicates that the experimental uncertainty was correctly estimated. Table 1 gives the rotational and centrifugal distortion parameters.

The second studied sample contained the three isotopologues $\mathrm{CHDOH}-\mathrm{CHO}, \mathrm{CH}_{2} \mathrm{OH}-\mathrm{CDO}$, and $\mathrm{CHDOH}-\mathrm{CDO}$ with an abundance ratio of $2: 1: 1$. The analysis was carried out for each isotopologue independently, as previously described for $\mathrm{CH}_{2} \mathrm{ODCHO}$, and all newly assigned lines are $b$-type transitions, too. However, the analysis was complicated by the presence of three different isotopologues and the presence of many pyridine lines resulting from the synthesis procedure.

- For CHDOH-CHO a total of 945 new lines were identified (1340 transitions because there are 179 unresolved doublets and 72 quadruplets) up to $J=64$ and $K_{a}^{\prime}=27$.

- For CHDOH-CDO we obtained 916 new identified lines (1368 transitions besauce there are 197 unresolved doublets and 85 quadruplets) up to $J=67$ and $K_{a}^{\prime}=29$.

- Finally for $\mathrm{CH}_{2} \mathrm{OH}-\mathrm{CDO}$ we obtained 858 new identified lines (1268 transitions because there are 203 unresolved doublets and 69 quadruplets) up to $J=65$ and $K_{a}^{\prime}=24$.

Table 1 gives the rotational and centrifugal distortion parameters determined for these three other isotopologues. We used the same parameter set for each isotopologue that was determined 
Table 1. Determined rotational and centrifugal distortion parameters.

\begin{tabular}{lcccc}
\hline \hline Parameters & $\mathrm{CH}_{2}$ OD-CHO & CHDOH-CHO & CHDOH-CDO & $\mathrm{CH}_{2}$ OH-CDO \\
\hline $\mathrm{A} / \mathrm{MHz}$ & $17490.68230(18)$ & $16987.80362(18)$ & $15862.45361(17)$ & $17150.99706(21)$ \\
$\mathrm{B} / \mathrm{MHz}$ & $6499.72649(12)$ & $6385.430683(91)$ & $6233.230843(90)$ & $6362.87482(10)$ \\
$\mathrm{C} / \mathrm{MHz}$ & $4882.948293(76)$ & $4843.811766(70)$ & $4663.584048(65)$ & $4778.922377(73)$ \\
$\Delta_{J} / \mathrm{kHz}$ & $6.349256(81)$ & $5.622519(55)$ & $4.988629(52)$ & $5.471924(59)$ \\
$\Delta_{J K} / \mathrm{kHz}$ & $-17.93851(31)$ & $-15.51877(26)$ & $-13.56931(22)$ & $-16.70029(31)$ \\
$\Delta_{K} / \mathrm{kHz}$ & $37.67004(34)$ & $35.90235(46)$ & $28.82809(37)$ & $36.52446(81)$ \\
$\delta_{J} / \mathrm{kHz}$ & $1.898798(40)$ & $1.656979(23)$ & $1.508496(23)$ & $1.650572(26)$ \\
$\delta_{K} / \mathrm{kHz}$ & $8.81685(86)$ & $7.59317(44)$ & $6.10186(36)$ & $7.30024(46)$ \\
$\Phi_{J} / \mathrm{mHz}$ & $-0.008080(30)$ & $-0.006612(17)$ & $-0.005815(16)$ & $-0.006307(18)$ \\
$\Phi_{J K} / \mathrm{Hz}$ & $0.15452(42)$ & $0.13274(27)$ & $0.10725(21)$ & $0.12995(28)$ \\
$\Phi_{K J} / \mathrm{Hz}$ & $-0.6494(15)$ & $-0.5728(10)$ & $-0.43733(80)$ & $-0.5700(10)$ \\
$\Phi_{K} / \mathrm{Hz}$ & $0.7573(12)$ & $0.71373(86)$ & $0.50818(67)$ & $0.7048(12)$ \\
$\phi_{J} / \mathrm{mHz}$ & $-0.002662(15)$ & $-0.0022434(84)$ & $-0.0020597(82)$ & $-0.0021147(88)$ \\
$\phi_{J K} / \mathrm{mHz}$ & $-0.03007(40)$ & $-0.02255(23)$ & $-0.01534(19)$ & $-0.01999(22)$ \\
$\phi_{K} / \mathrm{Hz}$ & $0.2636(47)$ & $0.1945(29)$ & $0.1254(21)$ & $0.1777(26)$ \\
& & & & \\
$J_{\mathrm{MAX}}, K_{a \mathrm{MAX}}$ & 64,37 & 64,27 & 67,29 & 65,24 \\
Freq & 626777.73 & 629356.35 & 626675.43 & 623233.58 \\
nbr of transitions & 889 & 945 & 916 & 858 \\
$\sigma_{\mathrm{FIT}} / \mathrm{kHz}$ & 27 & 26 & 26 & 27 \\
\hline
\end{tabular}

Table 2. Assigned transitions for the ground state of $\mathrm{CH}_{2} \mathrm{OD}-\mathrm{CHO}$.

\begin{tabular}{cccccccccc}
\hline \hline$J^{\prime}$ & $K_{a}^{\prime}$ & $K_{c}^{\prime}$ & $J^{\prime \prime}$ & $K_{a}^{\prime \prime}$ & $K_{c}^{\prime \prime}$ & $\begin{array}{c}\text { Frequency } \\
(\mathrm{MHz})\end{array}$ & $\begin{array}{c}\text { Obs. }- \text { Calc. } \\
(\mathrm{MHz})\end{array}$ & $\begin{array}{c}\text { Uncertainty } \\
(\mathrm{MHz})\end{array}$ & $\begin{array}{c}\text { Intensity weighting } \\
\text { for blended lines }\end{array}$ \\
\hline 25 & 11 & 14 & 25 & 10 & 15 & 242089.1640 & -0.0346 & 0.0300 & $\ldots$ \\
25 & 11 & 15 & 25 & 10 & 16 & 242098.7040 & -0.0043 & 0.0300 & $\ldots$ \\
24 & 11 & 13 & 24 & 10 & 14 & 242884.5120 & 0.0257 & 0.0300 & $\cdots$ \\
24 & 11 & 14 & 24 & 10 & 15 & 242888.6880 & -0.0039 & 0.0300 & $\cdots$ \\
23 & 11 & 12 & 23 & 10 & 13 & 243577.9080 & 0.0077 & 0.0300 & $\ldots$ \\
23 & 11 & 13 & 23 & 10 & 14 & 243579.6720 & -0.0094 & 0.0300 & $\cdots$ \\
22 & 11 & 11 & 22 & 10 & 12 & 244180.5840 & -0.0272 & 0.0300 & $\cdots$ \\
22 & 11 & 12 & 22 & 10 & 13 & 244181.3400 & 0.0094 & 0.0300 & $\ldots$ \\
21 & 11 & 10 & 21 & 10 & 11 & 244702.4760 & 0.0192 & 0.0300 & $5.0 \mathrm{E}-01$ \\
21 & 11 & 11 & 21 & 10 & 12 & 244702.4760 & 0.0192 & 0.0300 & $5.0 \mathrm{E}-01$ \\
20 & 11 & 9 & 20 & 10 & 10 & 245151.7200 & 0.0551 & 0.0300 & $5.0 \mathrm{E}-01$ \\
20 & 11 & 10 & 20 & 10 & 11 & 245151.7200 & 0.0551 & 0.0300 & $5.0 \mathrm{E}-01$ \\
\hline
\end{tabular}

Notes. This table and those of other isotopologues are available in their entirety at the CDS. A portion is shown here for guidance regarding its form and content.

by trial as best (i.e., lowest rms with minimal number of parameters). All quartic and sextic centrifugal terms were determined. The uncertainties on the parameters and the weighted rms of the fits $(0.87,0.87$ and 0.90 for $\mathrm{CHDOH}-\mathrm{CHO}, \mathrm{CHDOH}-\mathrm{CDO}$, $\mathrm{CH}_{2} \mathrm{OH}-\mathrm{CDO}$ respectively) are similar for all isotopologues.

A full list of measurements is given in Tables 2-5 for $\mathrm{CH}_{2} \mathrm{OD}-\mathrm{CHO}, \mathrm{CHDOH}-\mathrm{CHO}, \mathrm{CH}_{2} \mathrm{OH}-\mathrm{CDO}$, and $\mathrm{CHDOH}-$ $\mathrm{CDO}$, respectively. These tables give the rotational quantum numbers, the observed frequencies, the residuals (obs-cal), and the assumed experimental uncertainties. Blended transitions are treated by fitting the intensity-averaged frequency, and this weighting is also given in the tables. Lines with obvious experimental problems are not included ${ }^{2}$.

\section{Conclusion}

The rotational spectra of three mono-deuterated glycolaldehyde isotopologues and a doubly deuterated one in the ground vibrational state have been characterised up to $630 \mathrm{GHz}$. This

\footnotetext{
2 Only the first 12 lines of Table 2 appear in the paper edition, a complete version of all species is available at the CDS.
}

frequency range is appropriate for a first astronomical identification. Most importantly for an astrophysical research, the new measurements provide complete and precise predictions in the range of one to several hundred $\mathrm{GHz}$, which is the optimum range for detection, taking into account the supposed temperature ( $\geq 35 \mathrm{~K}$ see introduction) and instrumental sensitivity. It is essential to be able to search for transitions over a wide frequency range not only to confirm the detection, but also because of spectral cluttering from other species. The measurements at higher frequency ensure the reliabilty of the predictions, but may also be useful in their own right, especially if glycolaldeyde should be detected at higher temperatures. The increased sensitivity provided by a new generation of radio-astronomical instruments (e.g., upgrading at the IRAM-30 m) and by new facilities such as the ALMA interferometer renders the prospect of detecting new deuterated isotopologues in astrophysical environments a realistic one. Using the recently measured data, we have therefore started to search for deuterated glycolaldehyde in existing spectral surveys of hot-core sources and plan new observations.

Acknowledgements. This work is supported by the contract ANR-08-BLAN0225 and by the Programme National de Physico-Chimie du Milieu Interstellaire (PCMI-CNRS) and J.-C.G. thanks the Centre National d'Etudes Spatiales 
A. Bouchez et al.: The submillimeter spectrum of deuterated glycolaldehydes

(CNES) for financial support. We would like to thank the referee for his/her useful comments about the fits.

\section{References}

Beltrán, M. T., Cesaroni, R., Neri, R., et al. 2005, A\&A, 435, 901

Beltrán, M. T., Codella, C., Viti, S., Neri, R., \& Cesaroni, R. 2009, ApJ, 690, L93

Bennett, C. J., \& Kaiser, R. I. 2007, ApJ, 661, 899

Butler, R. A. H., De Lucia, F. C., Petkie, D. T., et al. 2001, ApJS, 134, 319

Carroll, P. B., Drouin, B. J., \& Widicus Weaver, S. L. 2010, ApJ, 723, 845

Cazaux, S., Caselli, P., \& Spaans, M. 2011, ApJ, 741, L34

Ceccarelli, C., Castets, A., Loinard, L., Caux, E., \& Tielens, A. G. G. M. 1998, A\&A, 338, L43

Coutens, A., Vastel, C., Caux, E., et al. 2012, A\&A, 539, A132

Hollis, J. M., Lovas, F. J., \& Jewell, P. R. 2000, ApJ, 540, L107

Halfen, D. T., Apponi, A. J., Woolf, N., Polt, R., \& Ziurys, L. M. 2006, ApJ, 639, 237

Hamberg, M., Österdahl, F., Thomas, R. D., et al. 2010, A\&A, 514, A83

Jalbout, A. F., Abrell, L., Adamowicz, L., et al. 2007, Astrobiology, 7, 433
Lattelais, M., Pauzat, F., Ellinger, Y., \& Ceccarelli, C. 2009, ApJ, 696, L133 Margulès, L., Motiyenko, R. A., Alekseev, E. A., \& Demaison, J. 2010, J. Mol. Spectrosc., 260, 23

Marstokk, K., \& Møllendal, H. 1970, J. Mol. Struct., 5, 205

Marstokk, K., \& Møllendal, H 1971, J. Mol. Struct., 7, 101

Marstokk, K., \& Møllendal, H. 1973, J. Mol. Struct., 16, 259

Mauersberger, R., Henkel, C., Jacq, T., \& Walmsley, C. M. 1988, A\&A, 194, L1

Motiyenko, R. A., Margulès, L., Alekseev, E. A., Guillemin, J. C., \& Demaison, J. 2010, J. Mol. Spectrosc., 264, 94

Oliveira C., \& Hébrard G. 2006, ApJ, 653, 345

Parise, B., Ceccarelli, C., Tielens, A. G. G. M., et al. 2002 A\&A, 393, L49

Parise, B., Castets, A., Herbst, E., et al. 2004, A\&A, 416, 159

Pickett, H. M., Poynter, R. L., Cohen, E. A., et al. 1998, J. Quant. Spec. Radiat. Transf., 60, 883

Ratajczak, A., Taquet, V., Kahane, C., et al. 2011, A\&A, 528, L13

Simakov, A., Sekiguchi, O., Bunkan, A., Joakim C., \& Uggerud, E. 2011, J. Am. Chem. Soc, 133, 20816

Turner, B. E. 1990, ApJ, 362, L29

Widicus Weaver, S. L., Butler, R. A. H., Drouin, B. J., et al. 2005, ApJS, 158, 188 